\title{
Red Dwarfs as Sources of Cosmic Rays
}

\author{
Vera Yu. Sinitsyna ${ }^{1, *}$, Vera G. Sinitsyna ${ }^{1, * *}$, and Yurii I. Stozhkov ${ }^{1}$ \\ ${ }^{1}$ P.N. Lebedev Physical Institute, Moscow, Russia
}

\begin{abstract}
The Pamela, Fermi, AMS-02 spectrometer experimental data cannot be explained using the diffusive models of propagation of cosmic-rays accelerated at the supernova shocks and require the existence of nearby sources of cosmic rays at the distances less than one kpc. These sources could explain the growth of the ratio of galactic positrons to electrons with an energy increase, the complex dependence of the exponent of the proton and alpha spectra on the energy, the cosmic ray anomaly component origin. We consider active dwarf stars as possible sources of galactic cosmic rays in the energy range up to $\sim 10^{14} \mathrm{eV}$. The generation of high-energy cosmic rays should be accompanied by highenergy gamma-ray emission, which may be detected. The TeV gamma-ray emission mostly of flaring type from active red dwarf stars V388 Cas, V547 Cas, V780 Tau, V962 Tau, V1589 Cyg, GJ 3684, GJ 1078 and GL 851.1 was detected recently with SHALON long-term observations. This result confirms that active dwarf stars are also the sources of high-energy galactic cosmic rays.
\end{abstract}

\section{Introduction}

Supernova explosions are considered as sources of Galactic cosmic rays. Indeed, investigations of last years show that the Supernova Remnants (SNRs) are powerful accelerators of cosmic-ray particles up to $\sim 10^{17} \mathrm{eV}$. But, the proton and helium spectra at high and very high energies measured by PAMELA and AMS-02 can't be explained using the diffusive models of propagation of supernova shock born cosmic-rays. Also, there is no explanation of the excess of 10 - $300 \mathrm{GeV}$ positron flux detected by PAMELA and AMS-02 experiments (Fig. 1). An interpretation of all these observations hints at a presence of nearby sources of cosmic rays at the distances less than $1 \mathrm{kpc}$ from the solar system. We consider active dwarf stars as possible sources of galactic cosmic rays with energies up to $\sim 10^{14} \mathrm{eV}$ whose generation is accompanied by the $\mathrm{X}$-ray and high-energy $\gamma$-ray emission including $\mathrm{TeV}$-energy range.

\section{On red dwarf stars}

The dwarf stars belong to the $\mathrm{G}$ - M classes of the main sequence of stars and are more than $90 \%$ of all $2 \times 10^{11}$ stars in our Galaxy. The nearest of the dwarf stars are at several parsecs from Earth and are uniformly distributed inside of the galactic disc [1]. These stars produce powerful stellar flares, which sometimes occur several times per day, but its flare frequency is uncertain. The total energy release estimates as $10^{34}-10^{36}$ ergs $[1,2]$. The total energy of cosmic rays produced by the dwarf star flares in the Galaxy is estimated as $\approx 10^{51}-10^{53} \mathrm{ergs}$ [3]. This could provide the amount of energy of charged cosmic rays in our Galaxy compared with ones from SN explosions. If active dwarf stars produce cosmic rays with $E \leq 10^{14} \mathrm{eV}$, it can explain the anomalies detected by PAMELA, AMS-02, CALET, DAMPE [4, 5].

\footnotetext{
*e-mail: sinicinavy@lebedev.ru

**e-mail: sinits@sci.lebedev.ru
} 


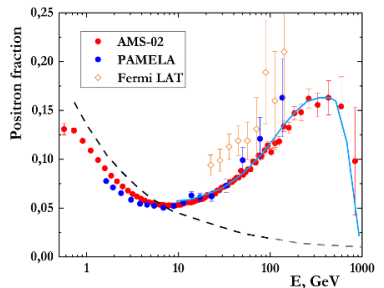
Figure 1. The ratio of $e^{+}$flux to the sum of $e^{-}$and $e^{+}$fluxes versus energy of these particles. Blue points are the PAMELA data and red points are the AMS-02 data. Dashed line shows model predictions from [10]. Blue line shows the result of calculations of ratio fluxes of $e^{+}$to $\left(e^{+}+e^{-}\right)$versus energy if the probability of positron release from stellar flare site is taken into account.

\section{Very high energy emission of red dwarf stars}

$\mathrm{TeV} \gamma$-ray emission from the M-stars was detected in SHALON experiment [3] on the level of $>5 \sigma$ and the average fluxes shown in Table 1. During the long-term observations of red dwarfs the flux variations of at energies up to $10 \mathrm{TeV}$ were detected. Both, average $(\triangle)$ and flaring ( $\Delta$ ) spectral energy distributions (SED) are presented in Fig. 2. The energy spectrum shapes at $E>800 \mathrm{GeV}$ can be fitted with a soft power law: $d F / d E \propto E^{k}$, the details are listed in Table 1. The duration of high flux periods corresponding to the flare spectra was found to befrom 2 to 5 days [3]. The detected $\mathrm{TeV} \gamma$-ray fluxes show that these objects produce flares with a total energy of $\sim 10^{34}-10^{35} \mathrm{ergs}$. But, the most powerful solar flare is $10^{32} \mathrm{ergs}$.

The searches for counterparts for the detected at TeV energy M-stars in X-rays and MeV$\mathrm{GeV}$ energies were performed. X-ray emission from the position of these objects was detected by ROSAT . In Fig. 2 the emission maps of listed red dwarfs by SHALON in comparison with data from X-ray data are presented. The X-ray emission $(0.1-2.4 \mathrm{keV})$ from the position of discussed red dwarfs (error $\sim 10^{\prime \prime}$ ) was detected with ROSAT with energy fluxes from $3.9 \times 10^{-13}$ to $2.5 \times 10^{-12} \mathrm{erg} \mathrm{cm}^{-2} \mathrm{~s}^{-1}$ and indexes of $\sim-2$. It corresponds to the estimated luminosity of from $5 \times 10^{27}$ to $1 \times 10^{29} \mathrm{erg} \mathrm{s}^{-1}$. High-energy emission from unassociated sources at distances of $<0.6^{\circ}$ from V962 Tau, V1589 Cyg, GJ 1078 and GL 851.1 were also detected by Fermi LAT. The spectra of each of unassociated objects are presented in Fig. 2 with bow tie and is consistent with SHALON data for these red dwarfs at TeV energies. Unfortunately, V388 Cas, V547 Cas, V780 Tau and GJ 3684 were not active enough after 2008 to produce a significant detection by Fermi LAT launched in 2008. But, V1589 Cyg, V962 Tau, GJ 1078, GL 851.1 were quite active during the whole period of observations and the nearby Fermi LAT unassociated sources may actually be these red dwarfs.

\section{Discussion}

The discovery of flaring red dwarf stars at $E \approx 1 \div 20 \mathrm{TeV}$ by SHALON, confirms, that these stars are also the sources of high energy cosmic rays and can explain a number phenomenon viewed in space experiments. The space experiments in the energy range from $\sim 50 \mathrm{GeV}$ up to several hundreds of $\mathrm{GeV}$ show that the spectra of protons and $\alpha$-particles do not follow single power law with constant exponent $\gamma[5,7]$. If galactic cosmic rays are produced by shock-waves from supernova explosions and cosmic ray life-time in Galaxy is about $10^{6}-10^{8}$ years the diffusion process smooths the spectra of these particles. As there are many active dwarf stars nearby the solar system and their stellar activity is quasi-periodic, one can expect

Table 1. Red stars of Flare star catalogue [1] viewed by SHALON.

Here ${ }^{a}$ Integral flux at energy $>800 \mathrm{GeV}$ in units of $10^{-12} \mathrm{~cm}^{-2} \mathrm{~s}^{-1}$ and

${ }^{b}$ Index in fitting of TeV differential spectrum in form of power low $d F / d E \propto E^{k_{\gamma}}$ and $d F / d E \propto E^{k_{\text {Flare }}}$ )

\begin{tabular}{cccccc}
\hline Source & $\begin{array}{c}\text { Distance, } \\
\text { pc }\end{array}$ & $\begin{array}{c}\text { Spectral } \\
\text { type }\end{array}$ & $F(>0.8 \mathrm{TeV})^{a}$ & $k_{\gamma}^{b}$ & $k_{\text {Flare }}^{b}$ \\
\hline V388 Cas & 10.5 & M5V & $0.84 \pm 0.20$ & $-2.51 \pm 0.15$ & $-2.91 \pm 0.18$ \\
V547 Cas & 10.1 & dM2.5e/M2V & $0.14 \pm 0.03$ & $-2.41 \pm 0.29$ & $-2.12 \pm 0.32$ \\
V780 Tau & 10.4 & m/M5.5 or M7V & $0.23 \pm 0.03$ & $-2.52 \pm 0.15$ & $-3.01 \pm 0.21$ \\
V962 Tau & - & - & $0.39 \pm 0.04$ & $-2.54 \pm 0.16$ & $-2.95 \pm 0.22$ \\
V1589 Cyg & $23-32$ & dM4.5e & $0.13 \pm 0.02$ & $-2.91 \pm 0.18$ & $-3.15 \pm 0.29$ \\
GJ 1078 & 20.6 & M4.5V & $0.38 \pm 0.14$ & $-2.46 \pm 0.48$ & $-3.11 \pm 0.49$ \\
GJ 3684 & 16.5 & m/M4V & $0.33 \pm 0.11$ & $-2.64 \pm 0.27$ & $-3.04 \pm 0.26$ \\
GL 851.1 & 21.7 & dM0e or K5V & $0.55 \pm 0.09$ & $-2.54 \pm 0.42$ & $-2.32 \pm 0.21$ \\
\hline
\end{tabular}



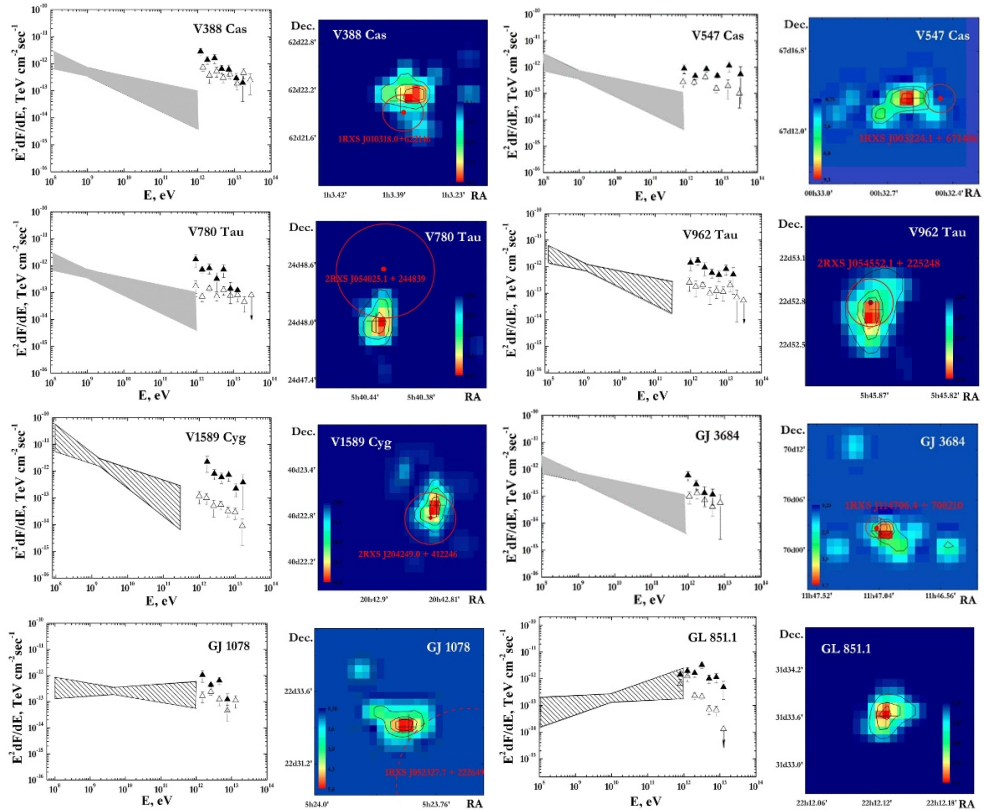

Figure 2. SEDs of red dwarfs by SHALON and Fermi LAT; Emission maps of red dwarfs by SHALON. that the value of exponent $\gamma$ may change if one or several such stellar objects are at the distances of several pc from the Sun.

Another problem could be explained with nearby cosmic ray sources is so-called anomaly component consisting of single ionized atoms like $\mathrm{He}, \mathrm{N}, \mathrm{O}, \mathrm{Ne}, \mathrm{C}, \mathrm{Mg}, \mathrm{Si}$ and others $[8,9]$ with energies from $\sim 5$ to $100 \mathrm{MeV} /$ nucleon. The anomaly component has a positive radial gradient in the heliosphere and undergoes a solar modulation. The mechanism of anomaly component production was proposed in [9] but the spacecraft Voyager-1, 2 did not confirm it. These single ionized atoms could be formed in flares of active dwarf stars if they are near the solar system. These single ionized atoms will be totally stripped if the ionization crosssection of $\sigma \approx 10^{-18} \mathrm{~cm}^{2}$ and the amount of interstellar matter between their source and the solar system is $\geq 1$. The interstellar medium density near Sun is about $\rho \sim 0.02$ atom $/ \mathrm{cm}^{3}$. Thus, the distance between the source and the solar system is $L \leq 1 /(\rho \sigma)$, i.e. $L \leq 30 \mathrm{pc}$, and there are about 560 flaring red dwarf stars within the sphere of the $\sim 30 \mathrm{pc}$ radius.

An important effect, discovered by the PAMELA and confirmed by AMS-02, is positron excess or unexpected positron fraction raise instead of decrease [10]. The observed positron fraction is starting to increase at $\sim 5 \mathrm{GeV}$ and then monotonically grows up to $\sim 350 \mathrm{GeV}$, then at energies $>500 \mathrm{GeV}$ it falls down. A possible interpretation of this effect may involve the active dwarf stars as the sources of cosmic rays up to $E \sim 10^{13} \mathrm{eV}$ [6] This new type sources of cosmic rays provides essential addition positron flux to one produced in the interstellar medium in interactions of cosmic rays with interstellar matter. (see line in Fig. 1).

\section{References}

[1] R.E. Gershberg et al., Astron. Astrophys. Suppl. Ser. 139, 555 (1999)

[2] S. Ohm \& C. Hoischen, MNRAS 474, 1335-1341 (2018)

[3] V.G. Sinitsyna, V.Y. Sinitsyna, Y.I.Stozhkov, Adv. Space Res. 64(12), 2585-2594 (2019)

[4] O. Adriani, G.C. Barbarino, G.A. Bazilevskaya et al., Nature 458(7238), 607-609 (2009)

[5] M. Aguilar, D. Aisa, B. Alpat et al., Phys. Rev. Lett. 114, 171103 (2015)

[6] Yu.I. Stozhkov, Bull. of RAS ser. Physics 75(3), 323-326 (2011)

[7] O. Adriani et al., Rivista del Nuovo Cimento 40(10), 473-522 (2017)

[8] M. Garcia-Munoz, G.M. Mason, J.A. Simpson, Astrophys. Journ. Lett. 182, L81 (1973)

[9] L. Fisk, B. Kozlovsky, R. Ramaty, Astrophys. Journ. Lett. 190, L35 (1974)

[10] I.V. Moskalenko \& A.W. Strong, Astrophys. J. 493, 694-707 (1998) 